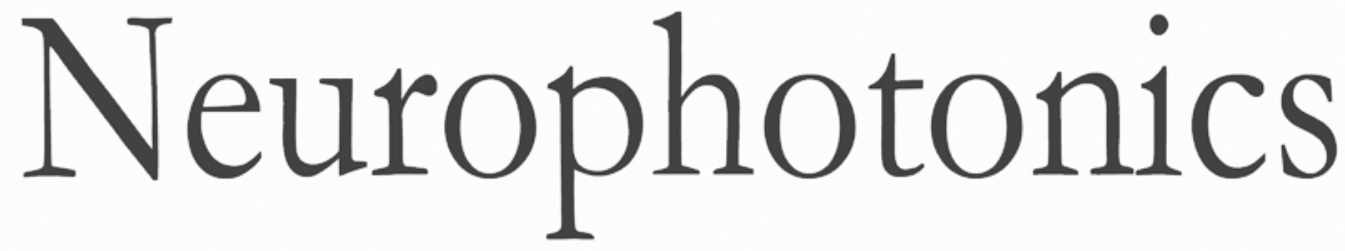

\title{
Impact of atherosclerotic disease on cerebral microvasculature and tissue oxygenation in awake LDLR-/-hApoB+/+ transgenic mice
}

\author{
Yuankang Lu \\ Cong Zhang \\ Xuecong Lu \\ Mohammad Moeini \\ Eric Thorin \\ Frédéric Lesage
}




\title{
Impact of atherosclerotic disease on cerebral microvasculature and tissue oxygenation in awake LDLR-I-hApoB+/+ transgenic mice
}

\author{
Yuankang Lu, ${ }^{a}$ Cong Zhang, ${ }^{b}$ Xuecong Lu, ${ }^{a}$ Mohammad Moeini, ${ }^{c}$ Eric Thorin,, and Frédéric Lesage ${ }^{a, b, \star}$ \\ aÉcole Polytechnique de Montréal, Laboratoire d'Imagerie optique et moléculaire, Montréal, Québec, Canada \\ 'Institut de Cardiologie de Montréal, Montréal, Québec, Canada \\ ${ }^{c}$ Amirkabir University of Technology (Tehran Polytechnic), Biomedical Engineering Department, Tehran, Iran \\ 'Université de Montréal, Department of Pharmacology and Physiology, Faculty of Medicine, Montréal, Québec, Canada
}

\begin{abstract}
We explore cortical microvasculature changes during the progression of atherosclerosis using young and old transgenic atherosclerotic (ATX) mice with thinned-skull cranial window. In awake animals, exploiting intrinsic signal optical imaging, Doppler optical coherence tomography, and two-photon microscopy, we investigate how the progression of atherosclerotic disease affects the morphology and function of cortical microvasculature as well as baseline cerebral tissue oxygenation. Results show that aged ATX mice exhibited weaker hemodynamic response in the somatosensory cortex to whisker stimulation and that the diameter of their descending arterioles and associated mean blood flow decreased significantly compared with the young ATX group. Data from two-photon phosphorescence lifetime microscopy indicate that old ATX mice had lower and more heterogeneous partial pressure of oxygen $\left(\mathrm{PO}_{2}\right)$ in cortical tissue than young ATX mice. In addition, hypoxic micropockets in cortical tissue were found in old, but not young, ATX mice. Capillary red blood cell (RBC) flux, RBC velocity, RBC velocity heterogeneity, hematocrit, and diameter were also measured using line scans with two-photon fluorescence microscopy. When compared with the young group, RBC flux, velocity, and hematocrit decreased and RBC velocity heterogeneity increased in old ATX mice, presumably due to disturbed blood supply from arterioles that were affected by atherosclerosis. Finally, dilation of capillaries in old ATX mice was observed, which suggests that capillaries play an active role in compensating for an oxygen deficit in brain tissue. ( ) The Authors. Published by SPIE under a Creative Commons Attribution 4.0 Unported License. Distribution or reproduction of this work in whole or in part requires full attribution of the original publication, including its DOI. [DOI: 10.1117/1.NPh.6.4.045003]
\end{abstract}

Keywords: cortical microvasculature; intrinsic signal optical imaging; Doppler optical coherence tomography; two-photon microscopy. Paper 19051R received May 28, 2019; accepted for publication Sep. 17, 2019; published online Oct. 24, 2019.

\section{Introduction}

Ischemic cerebrovascular diseases (CVD) and their complications are one of the leading causes of morbidity and mortality worldwide. Ischemic cerebrovascular events, such as ischemic stroke and transient ischemic attack also frequently lead to vascular dementia and persistent cognitive impairment. ${ }^{1-4}$ Studies have shown that atherosclerotic disease often plays a causative role in the onset of the ischemic CVDs. ${ }^{5-7}$ It is well known that in the presence of elevated plasma low-density lipoprotein (LDL), plaques can build up inside extracranial and intracranial large arteries. As a consequence, the luminal stenosis reduces or even blocks blood supply to the brain, causing oxygen and energy substrate deficiency in cerebral tissue. ${ }^{8-12}$ The impact of atherosclerotic disease on cerebral small vessels, however, still remains to be fully elucidated. ${ }^{13-15}$

Clinical neuroimaging, such as magnetic resonance imaging and computed tomography, evaluates cerebral small vessel disease-related ischemic parenchymal lesions rather than alterations of the cerebral microvasculature itself due to the limited spatial resolution of the imaging techniques and the small size of vessels. ${ }^{16-19}$ High-resolution optical imaging techniques such as intrinsic signal optical imaging (ISOI), optical coherence

*Address all correspondence to Frédéric Lesage, E-mail: frederic.lesage@ polymtl.ca tomography (OCT), and two-photon microscopy combined with a genetically modified ATX mouse model thus provides an opportunity to investigate the effects of atherosclerotic disease on cerebral microvasculature. ${ }^{20-23}$ In this work, we used $\mathrm{LDLR}^{-/-} \mathrm{hApoB}^{+/+}$transgenic mice, which have LDL receptor deficiency and express the human apolipoprotein B-100 gene. Previous studies have reported that ATX mice at 3 months of age had no atherosclerotic lesions in the aorta, carotid arteries, or cerebral arteries, ${ }^{24-26}$ whereas mice at 6 months of age and older developed extensive atherosclerosis. Learning capacity and cognitive functions were also found to decline significantly in one-year-old ATX mice. ${ }^{25,27}$ Systemic chronic inflammation caused by atherosclerosis contributes to cerebral endothelial cell senescence and dysfunction, which further leads to blood-brain barrier (BBB) leakage and capillary microbleeding in ATX mice where atherosclerosis is fully developed. ${ }^{28}$

The objective of our study was to assess how cerebral hemodynamics, cerebral microvasculature, and cerebral tissue oxygenation were affected by hyperlipidemia-associated atherosclerotic disease. To achieve this goal, multiple optical imaging techniques were used, including ISOI, spectral-domain Doppler OCT, two-photon phosphorescence lifetime microscopy, and line scans with two-photon fluorescence excitation. ISOI was first used to evaluate the hemodynamic response in the somatosensory cortex to whisker stimulation. This parameter reflects the efficiency of blood flow regulation by cerebral vasculature to 
meet increased metabolic needs of the brain. ${ }^{29}$ Doppler OCT was then performed to investigate possible structural and functional changes of descending arterioles as well as ascending venules related to atherosclerotic disease. Cerebral tissue oxygenation, a crucial parameter for brain function, can be disturbed by inadequate blood flow in microvasculature. We measured absolute cerebral tissue $\mathrm{PO}_{2}$ using two-photon phosphorescence lifetime imaging coupled with the oxygen quenching phosphorescent dye PtP-C343 to assess the impact of changes in blood flow. Finally, we used two-photon fluorescence line scans to investigate the impact of atherosclerotic disease and its progression on capillary properties.

\section{Materials and Methods}

\subsection{Animal Preparation}

The procedures and protocols were approved by the Animal Research Ethics Committee of the Montreal Heart Institute, and all animal experiments were performed in accordance with the Canadian Council on Animal Care recommendations. The animal model that we used in our study was the $\mathrm{LDLR}^{-/-} \mathrm{hApoB}^{+/+}$transgenic mice. They exhibit markedly high concentration of plasma LDL and spontaneously develop atherosclerotic lesions on a chow diet after the age of 6 months. ${ }^{28,30-32}$ They were categorized into two age groups: young ATX mice (3-month old) and old ATX mice (12-month old). Our rationale in choosing the cut-off age of 12 months was as follows: ATX mice usually have shorter lifespans than wild type (WT) mice, thus making it difficult to study them at 24 months, which is typically considered to be old age in WT mice. Furthermore, confounding effects from other morbidity factors could become too important in 24-month-old ATX mice. In our previous work, ${ }^{28}$ where both ATX and WT mice at 12 months of age were used, we observed cognitive differences in the Morris Maze and a decrease in brain perfusion. It was also shown that endothelial dysfunction was already present in ATX mice at 12 months of age when compared with 3-month-old ATX mice. ${ }^{33}$ Thus the choice of 3-month-old and 12-month-old ATX mice allowed us to emphasize effects of atherosclerosis while minimizing the effects of aging (though present).

At 8 to 10 days before measurements, a thinned-skull cranial window of 3-mm diameter was prepared over the left barrel cortex, and a titanium head bar was attached to the mouse skull. A detailed description of the surgical procedure can be found in our previous work. ${ }^{22}$ During imaging sessions, the mouse head was fixed by the head bar onto a custom-built treadmill wheel, into which limbs were free to move to reduce stress. Four fixation training sessions were required prior to image acquisition to habituate the mice to head restraint. All measurements were made in awake mice to eliminate the influence of anesthesia on vascular and neural physiology. ${ }^{34-36}$

\subsection{Data Acquisition and Data Analysis}

\subsubsection{Intrinsic signal optical imaging}

ISOI was used to explore stimulus-evoked hemodynamic response. ISOI data were obtained from nine young ATX mice and eight old ATX mice using a home-built ISOI system. Mouse whiskers were deflected at a rate of $10 \mathrm{~Hz}$ by a piezo-based mechanical whisker stimulator to induce increased neural activity in the somatosensory cortex. Each stimulation period lasted $5 \mathrm{~s}$ followed by a 15 -s interstimulus interval and a randomized jitter interval of an average of $3 \mathrm{~s}$. Stimulation was repeated 10 times. The imaging system used green and red LEDs to illuminate the somatosensory cortex, and images were taken at a rate of $5 \mathrm{~Hz}$ for each color ( $10 \mathrm{~Hz}$ combined). Since oxygenated and deoxygenated haemoglobins have different extinction coefficients, relative changes in oxyhemoglobin $\left(\mathrm{HbO}_{2}\right)$, deoxyhemoglobin ( $\mathrm{HbR}$ ), and total hemoglobin ( $\mathrm{HbT}$ ) concentration in functional brain areas were calculated based on the images acquired with the two wavelengths following previous work. ${ }^{37}$ Animals were monitored for locomotion and whisker activity during acquisitions. In some acquisitions, stimulations induced movement of the animal. Thus multiple acquisitions were acquired and the ones minimizing movement were analyzed.

\subsubsection{Spectral-domain Doppler OCT}

A spectral-domain OCT operating in Doppler mode was used to image descending arterioles and ascending venules for which the direction of blood flow is mostly parallel to the light beam. Images were acquired from five young ATX mice and five old ATX mice with a home-built spectral-domain OCT system. The critical parts of the OCT system included superluminescent diode illumination (LS2000C, Thorlabs) with a center wavelength at $1310 \mathrm{~nm}$ and a bandwidth of $200 \mathrm{~nm}$, a custom-built spectrometer, a galvanometer scanner (GVS002, Thorlabs), and a $5 \times$ infinity corrected objective (Thorlabs). The axial and lateral resolution in biological tissue was $\sim 3.5$ and $\sim 8.5 \mu \mathrm{m}$, respectively. ${ }^{22}$ A $900 \times 900 \mu \mathrm{m}$ cortical area was scanned to get a 3-D Doppler OCT volume. The 3-D volume had 512 B-scans, and each B-scan was composed of 2048 A-lines. Adjacent A-lines were deliberately overlapped. The phase difference between two adjacent A-lines was computed to calculate the speed of blood flow in arterioles and venules. ${ }^{38}$ The A-line acquisition rate was set to $50 \mathrm{kHz}$, which enabled the system to detect blood flow speed between -13 and $13 \mathrm{~mm} / \mathrm{s}$ along the direction of the incident beam. The scan was repeated 10 times in the same volume to enhance the signal-to-noise ratio (SNR). Following 3-D Doppler volume reconstruction, arterioles and venules were segmented on en face slices at depths between 140 and $290 \mu \mathrm{m}$ beneath the cortical surface. Slices shallower than $140 \mu \mathrm{m}$ or deeper than $290 \mu \mathrm{m}$ were discarded to exclude the pial vessels and capillaries. Detected descending arterioles and ascending venules had an elliptical appearance on en face images. The vessel lumen diameter was estimated from the minor axis while the blood flow was calculated by multiplying the area of the ellipse by the blood flow velocity along the $z$ axis.

\subsubsection{Two-photon phosphorescence lifetime imaging}

A custom-built two-photon microscope was used for phosphorescence lifetime imaging. A detailed description of the imaging system can be found in our previous publication. ${ }^{22}$ Working with the PtP-C343 dye, phosphorescence lifetime imaging provided a way to quantify tissue oxygenation $\left(\mathrm{PO}_{2}\right) \cdot{ }^{39}$ Nine young ATX mice and seven old ATX mice were used in the measurement. Before $\mathrm{PO}_{2}$ measurement, the PtP-C343 dye solution $(\sim 150 \mu \mathrm{M}$ in ACSF) was injected into the mouse brain tissue through the thinned skull using a glass micropipette slowly pushed by a microsyringe pump injector (UMP3, World Precision). Meanwhile, a FITC-Dextran solution $(50 \mathrm{mg} / \mathrm{ml}$ in saline) was administered through the tail vein for cerebral 
angiography, which was used for the selection of region of interest (ROI) in phosphorescence lifetime imaging. 2-D $\mathrm{PO}_{2}$ grid measurements (20 pixels $\times 20$ pixels) were taken in ROIs (400 $\mu \mathrm{m} \times 400 \mu \mathrm{m})$ at different depths beneath the cortical surface. The phosphorescent dye at each location of the grid was excited by an $80-\mathrm{MHz}$ femtosecond laser oscillator gated on for $25 \mu \mathrm{s}$, and a $275-\mu$ s photon collection window was applied right after to obtain a time-resolved decay curve of the phosphorescent molecules. Absolute tissue $\mathrm{PO}_{2}$ was thus calculated based on the exponential decay rate, namely the lifetime. ${ }^{22}$ This excitation/relaxation cycle was repeated 3000 times for each location to increase the SNR. A baby monitor operating in the IR was used to monitor animal behavior during experiments. Further details about the imaging system components and the animal handling procedure can be found in Ref. 22 .

\subsubsection{Two-photon fluorescence line scans}

The same two-photon microscopy system was used for fluorescence line scans to investigate capillaries in seven young ATX mice and seven old ATX mice. In a separate session, FITCDextran solution $(50 \mathrm{mg} / \mathrm{ml}$ in saline) was administered by intravenous injection as a contrast agent to label blood plasma. We first scanned a cortical area of $100 \mu \mathrm{m} \times 100 \mu \mathrm{m}$ to obtain 2-D en face fluorescence intensity images in the $x-y$ plane. Once capillaries were identified, perpendicular and longitudinal scans of individual capillaries were conducted. Each line consisted of 100 sampling points and was scanned at a line rate of $800 \mathrm{~Hz} .200$ consecutive lines were stacked to form a spatiotemporal image of perpendicular or longitudinal scans [Figs. 4(a2) and 4(b2)]. RBCs appeared as dark streaks, whereas blood plasma appeared as bright streaks due to fluorescent FITC-Dextran solution. Parameters, including capillary diameter, RBC flux, RBC velocity, RBC velocity heterogeneity, and hematocrit, were retrieved from the perpendicular and longitudinal line-scan images. Capillary diameter was determined as the full-width at half-maximum of the fitted Gaussian curve from the spatiotemporal image of perpendicular line scans [Fig. 4(a2)]. RBC velocity is inversely proportional to the slope of the streaks on the spatiotemporal image of longitudinal line scans [Fig. 4(b2)]. ${ }^{40}$ RBC velocity heterogeneity was defined as the coefficient of variation of RBC velocity in every individual capillary. This parameter is assumed to be positively correlated with capillary transit time heterogeneity, since capillary transit time depends on RBC velocity. Capillary RBC flux was estimated by counting the number of RBCs passing by per second. Hematocrit in the investigated capillary was calculated using the equation, Hct $=\mathrm{RBC}$ flux $\times \mathrm{RBC}$ volume/capillary volumetric flow, with RBC volume estimated to be $55 \mu \mathrm{m}$. $^{3,22}$

\subsubsection{Statistics}

Data are presented as mean \pm standard error of the mean (SEM) when results from the two groups of ATX mice are shown in the figures. Statistical comparisons between the two groups were performed using unpaired two-sample $t$-tests in MATLAB (MathWorks). The null hypothesis that there was no difference between the two groups is rejected if $p$-value was $<0.01$. The number of the young and the old ATX mice used for each imaging session is indicated above.

\section{Results}

\subsection{Atherosclerosis is Associated with Reduced Hemodynamic Response}

The hemodynamic response in the somatosensory cortex following whisker stimulation was measured using ISOI. Figures 1(a)-1(c) show typical images of $\mathrm{HbO}_{2}, \mathrm{HbR}$, and $\mathrm{HbT}$ concentration changes due to stimulation. Images are shown at $3 \mathrm{~s}$ after the beginning of a whisker stimulation. $\mathrm{HbO}_{2}$, $\mathrm{HbR}$, and $\mathrm{HbT}$ concentration changes were averaged across the entire somatosensory cortex to get the time courses [Fig. 1(d)]. The peak value of the $\mathrm{HbO}_{2}$ and $\mathrm{HbT}$ time series and the inverted peak value of the HbR time series were used to quantify the hemodynamic response amplitude to whisker stimulation. As seen in Fig. 1(e), the old ATX group had significantly smaller amplitude for $\Delta \mathrm{HbO}_{2}, \Delta \mathrm{HbR}$, and $\Delta \mathrm{HbT}$ than the young ATX group. Hemodynamic responses were averaged to obtain group-averaged time evolutions of $\mathrm{HbO}_{2}$ and $\mathrm{HbR}$ [Fig. 1(f)]. The old ATX group showed a weaker response to whisker deflection than the young ATX group. Another observation was that the response undershoot in $\mathrm{HbO}_{2}$ was present in young mice but disappeared in older mice. However, no obvious difference was found in the time delay between the two groups.

\subsection{Atherosclerosis is Associated with Reduced Basal Blood Flow and Reduced Arteriolar but not Venular Diameter}

Using spectral-domain Doppler OCT, we investigated cerebral blood flow in single descending arterioles and ascending venules, which deliver oxygen-rich blood and collect oxygen-poor blood, respectively. Figure 2(a1) shows a typical en face Doppler OCT image, which was acquired at a depth of $180-\mu \mathrm{m}$ beneath the cortical surface. Detected arterioles and venules are encircled by red and blue lines in Fig. 2(a2). Vessel diameter, blood velocity, and blood flow for each identified vessel were calculated based on such segmented en face slices using a segmentation algorithm to avoid operator bias. The diameter and mean blood flow of descending arterioles in the old ATX mice decreased by $9.0 \%$ and $24.7 \%$, respectively, compared with the young ATX mice [Figs. 2(c) and 2(d)]. Arteriolar diameter and arteriolar mean blood flow showed a decreasing trend with the depth in both groups of ATX mice [Figs. 2(g) and 2(h)]. However, the old ATX group displayed significantly smaller arteriolar lumen size and arteriolar mean blood flow at all measured cortical depths when compared with the young ATX group, which suggests a systemic modulation to cerebral arterioles caused by atherosclerotic disease. Correlation between arteriolar mean blood flow and arteriolar diameter was examined using data obtained from both the young and the old ATX mice. A strong positive correlation was found with a correlation coefficient of 0.96 [Fig. 2(b)], suggesting that narrowed arterioles caused lower mean blood flow. Interestingly, for old ATX mice, arteriolar mean blood flow not only dropped more rapidly with depth than in the young ATX mice but saturated at a lower bound as if it reached a plateau. As depicted in Figs. 2(e) and 2(f)], both diameter and mean blood flow of ascending venules showed no difference between the two groups of ATX mice. 


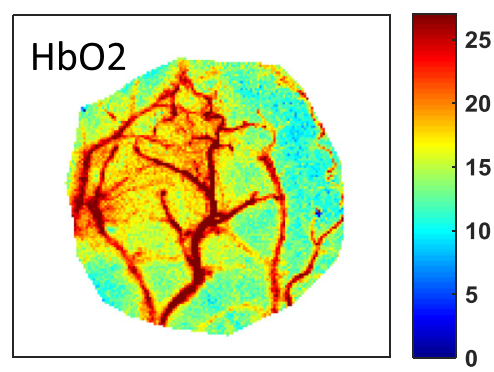

(a)

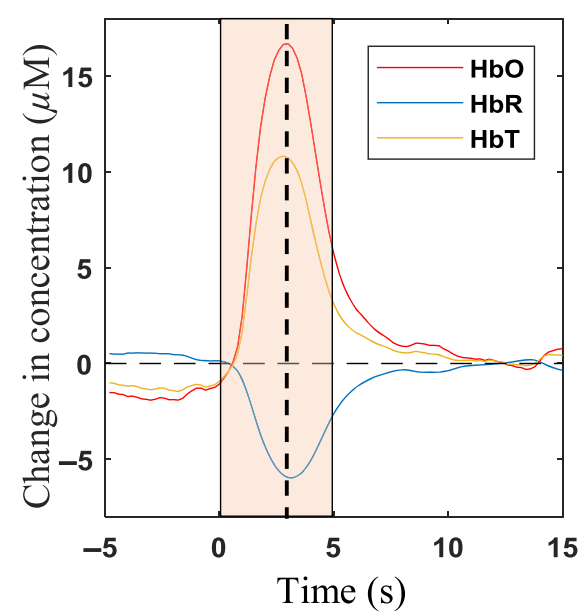

(d)

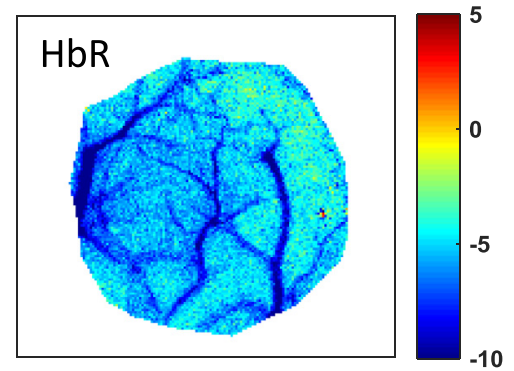

(b)

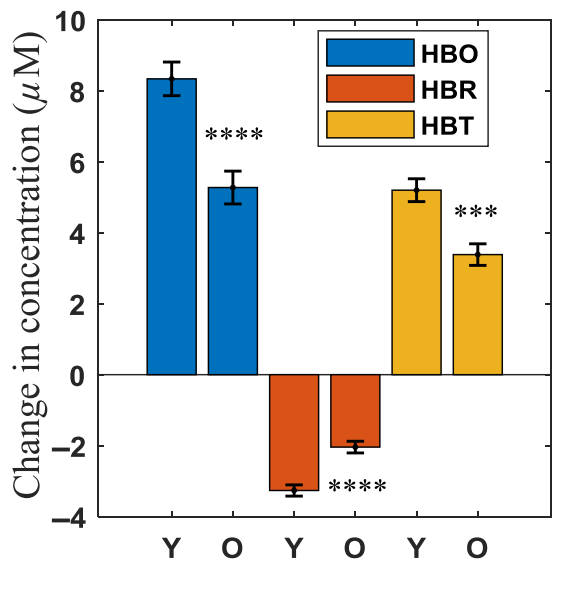

(e)

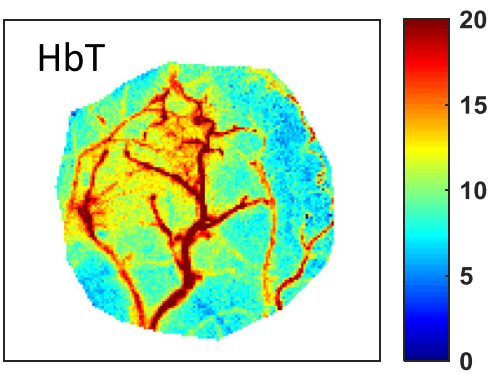

(c)

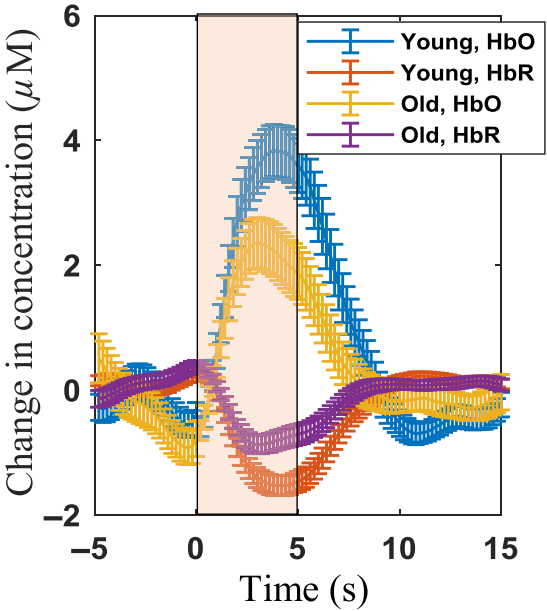

(f)

Fig. 1 ISOI used to measure hemodynamic response in somatosensory cortex following whisker stimulation. (a)-(c) Images of relative changes in $\mathrm{HbO}_{2}, \mathrm{HbR}$, and $\mathrm{HbT}$ acquired $3 \mathrm{~s}$ after the start of the stimulus. (d) A typical hemodynamic response obtained by averaging $\mathrm{HbO}_{2}, \mathrm{HbR}$, and $\mathrm{HbT}$ changes across the somatosensory cortex for a representative mouse. The transparent orange zone represents the 5-s whisker stimulation. The dashed black line corresponds to the moment when images (a)-(c) were acquired. (e) Comparison of the peak value of $\mathrm{HbO}_{2}, \mathrm{HbR}$, and $\mathrm{HbT}$ concentration changes after each stimulation. The young ATX mice had significantly higher peak values for $\Delta \mathrm{HbO}_{2}, \Delta \mathrm{HbR}$, and $\Delta \mathrm{HbT}$ than the old ATX mice. (f) Group-averaged time series of $\Delta \mathrm{HbO}_{2}$ and $\Delta \mathrm{HbR}$ for the young and the old ATX mice. Data are shown as mean \pm SEM. Unpaired $t$-test was performed: ${ }^{* \star \star} p<0.0001$ and ${ }^{\star \star \star} p<0.001$. $\mathrm{Y}$, young and $\mathrm{O}$, old.

\subsection{Reduced Blood Flow is Associated with Reduced Tissue $\mathrm{PO}_{2}$ and Pockets of Hypoxia}

Two-photon phosphorescence lifetime imaging was used to investigate whether reduced blood flow affected cerebral tissue oxygenation through impaired oxygen delivery from microvasculature. In each mouse, tissue $\mathrm{PO}_{2}$ data were acquired at six different depths ranging from 40 - to $180-\mu$ m beneath the cortical surface. Figure 3(b) shows the distribution of $\mathrm{PO}_{2}$ values for the young and the old ATX group. The distribution of tissue $\mathrm{PO}_{2}$ value for old ATX mice was shifted toward lower values when compared with young ATX mice. Focusing on the potential for hypoxia, we compared the frequency of samples with low $\mathrm{PO}_{2}$ value $(<15 \mathrm{mmHg})$ between the two groups [inset of Fig. 3(b)]. The percentage of low $\mathrm{PO}_{2}$ points changed from $7.7 \%$ in young ATX mice to $12.6 \%$ in old ATX ones. Overall, the mean value of tissue $\mathrm{PO}_{2}$ in the young ATX mice was $15 \%$ higher than that in the old ATX mice [Fig. 3(c)]. Moreover, the old ATX group showed significantly higher spatial heterogeneity of tissue oxygenation, suggesting capillary delivery heterogeneity [Fig. 3(d)]. The analysis of cerebral tissue $\mathrm{PO}_{2}$ versus depth (limited to the depth that was accessible in this thin-skull preparation) showed a decreasing trend in both ATX groups, but the young ATX group had significantly higher tissue $\mathrm{PO}_{2}$ at all depths [Fig. 3(e)]. The decrease of cerebral tissue $\mathrm{PO}_{2}$ and the increase of its spatial heterogeneity could cause low $\mathrm{PO}_{2}$ sites to colocate forming hypoxic micropockets. To look for the presence of hypoxic micropockets, we defined them as regions comprising at least 15 sampled points whose $\mathrm{PO}_{2}$ value is lower than 5 $\mathrm{mmHg}$. Figure 3(a2) shows an example of an interpolated tissue $\mathrm{PO}_{2}$ image with the presence of a hypoxic micropocket. In our study, hypoxic micropockets were found in two old ATX mice out of seven, whereas none of the nine ATX young mice had hypoxic micropockets.

\subsection{Cerebral Hypoperfusion and Decreased Tissue $\mathrm{PO}_{2}$ is Associated with Capillary Dilation}

To better understand how atherosclerotic disease affects cerebral microvasculature, we further investigated capillaries, which bridge between descending arterioles and cerebral tissue in terms of gas and substance exchange. Even though capillaries are not anticipated to be directly affected by atherosclerotic lesions because of their simple wall structure, high circulating plasma lipids and proinflammatory factors associated with atherosclerotic disease can still cause deleterious effects on 


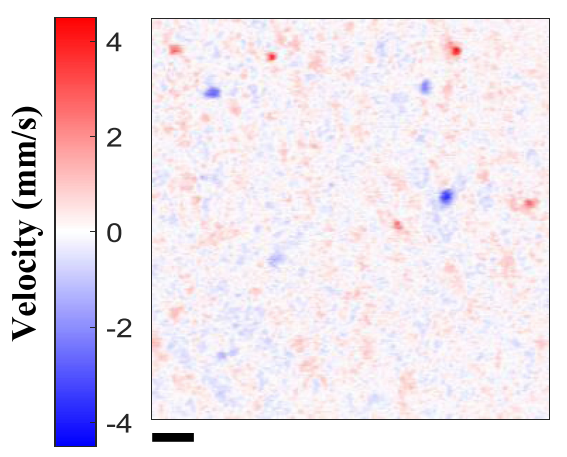

(a1)

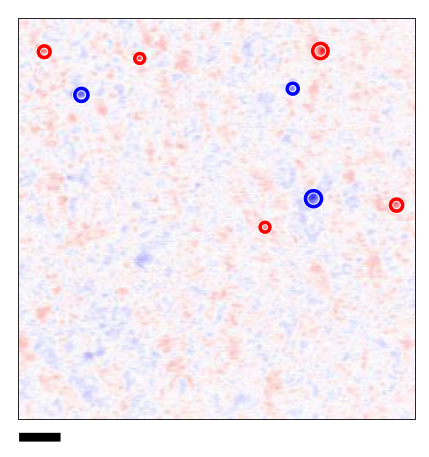

(a2)

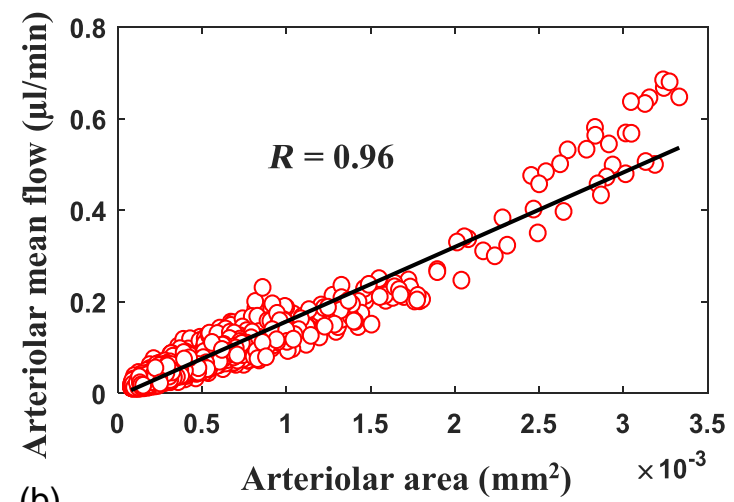

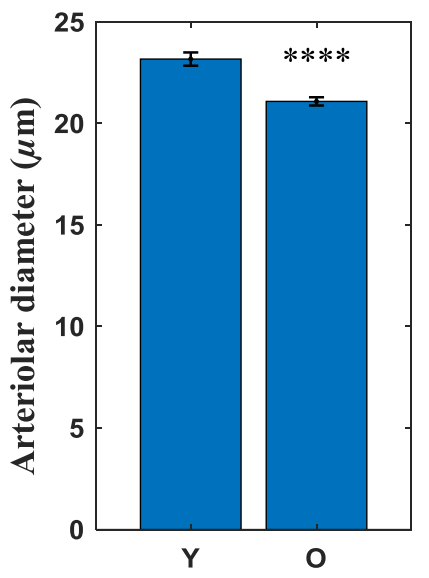

(c)

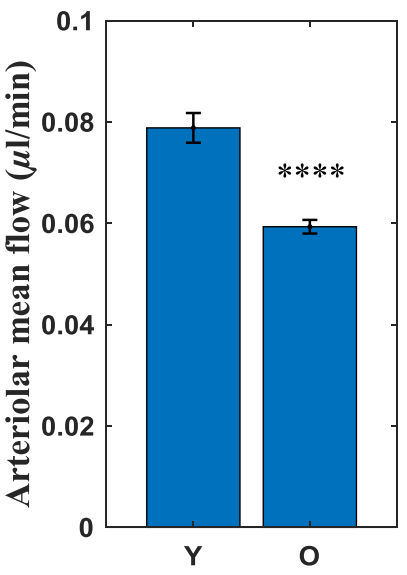

(d)

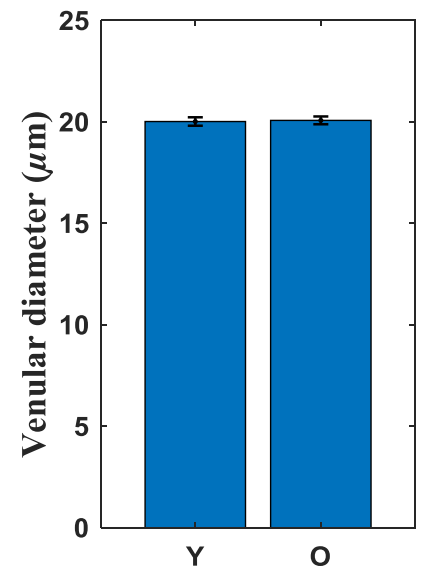

(e)

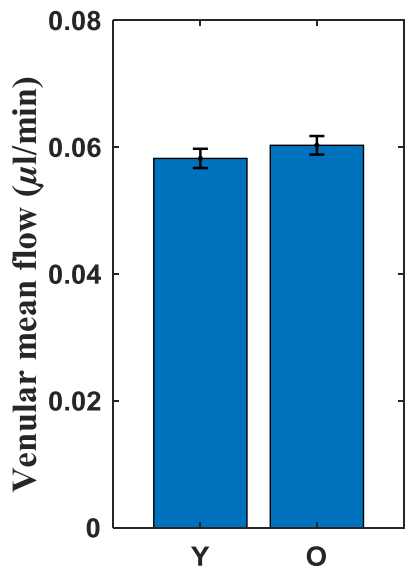

(f)
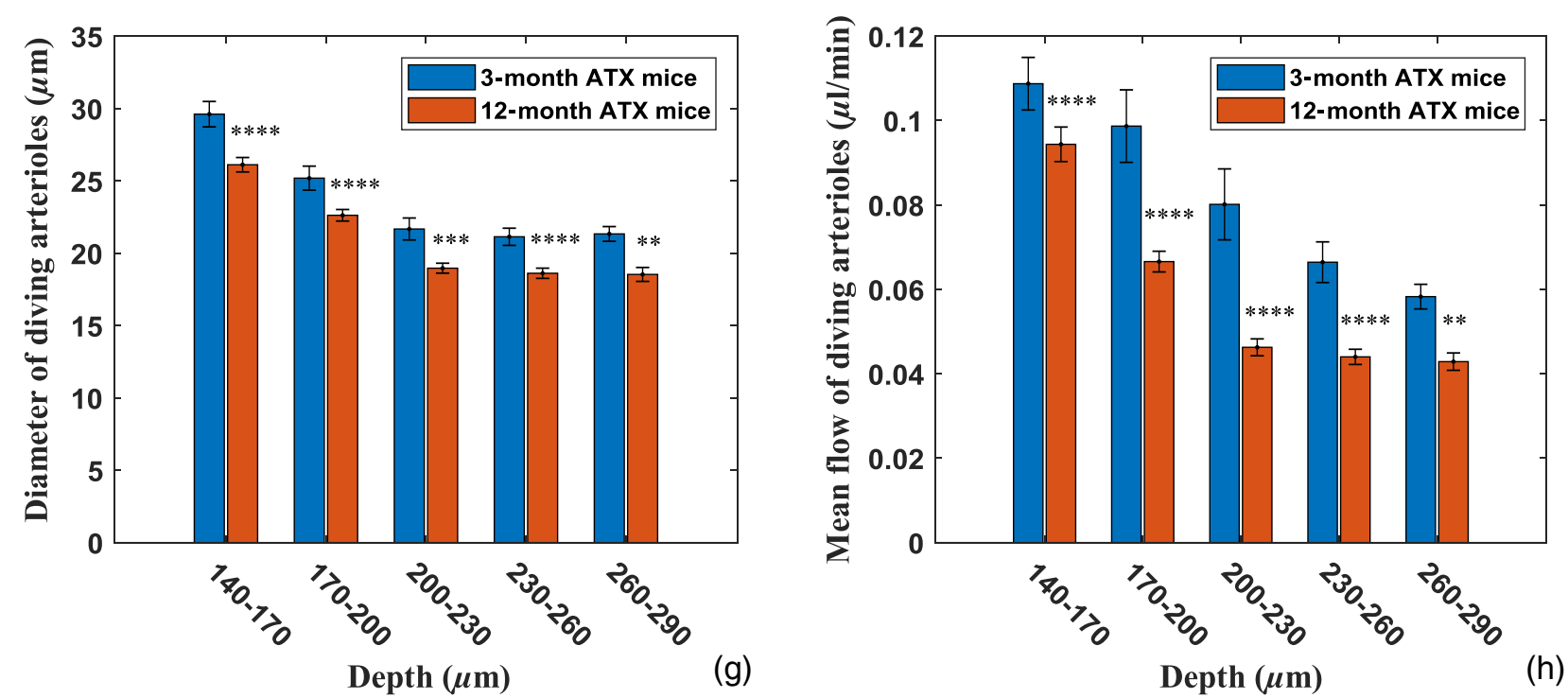

Fig. 2 Arteriolar and venular parameters obtained from Doppler OCT data. (a1) An en face Doppler OCT image obtained at a depth of $180-\mu \mathrm{m}$ beneath the cortical surface of a representative mouse. The scale bar is $100 \mu \mathrm{m}$. (a2) The same Doppler OCT image with identified arterioles and venules encircled by red and blue circles, respectively. (b) Correlation between arteriolar mean blood flow and arteriolar diameter. (c), (d) Comparison of arteriolar diameter and mean blood flow between the two ATX groups. The young ATX mice had significantly larger arteriolar diameter and significantly higher arteriolar mean blood flow than the old ATX mice. (e), (f) Comparison of venular diameter and mean blood flow between the two groups of ATX mice. No significant difference was found for venules between the two groups. (g), (h) The diameter and the mean blood flow of descending arterioles at five different depths for the young and the old ATX mice. Arteriolar diameter and mean blood flow at all range of depths decreased significantly in the old ATX mice when compared with the young ATX mice. Data are shown as mean \pm SEM. Unpaired $t$-test was performed: ${ }^{\star \star \star \star} p<0.0001,{ }^{\star \star \star} p<0.001$, and ${ }^{\star \star} p<0.01$. Y, young and O, old. 


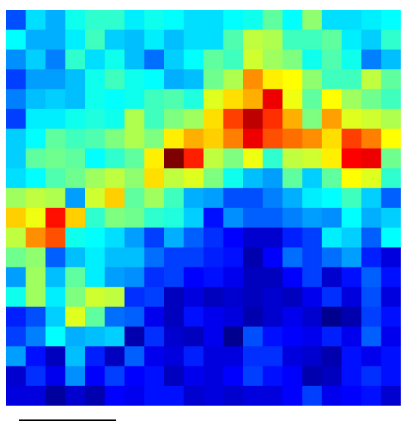

(a1)

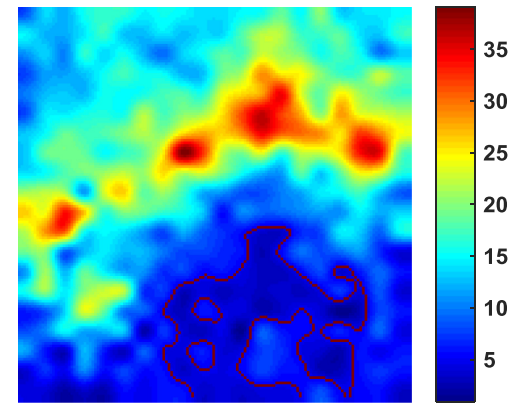

(a2)

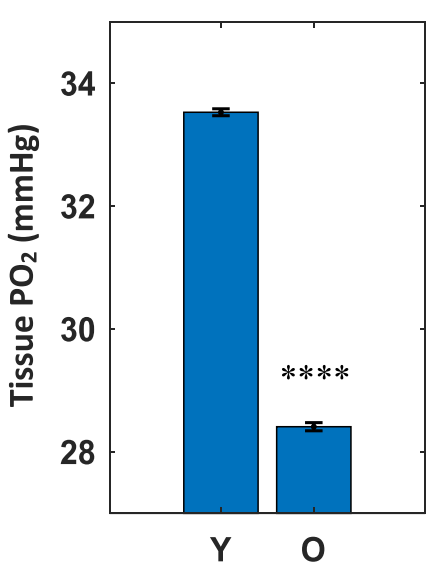

(c)

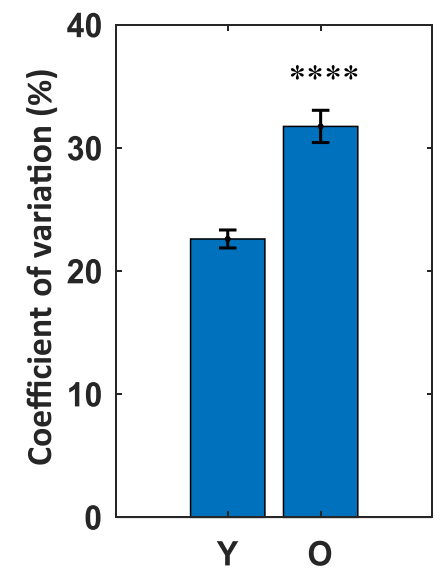

(d)
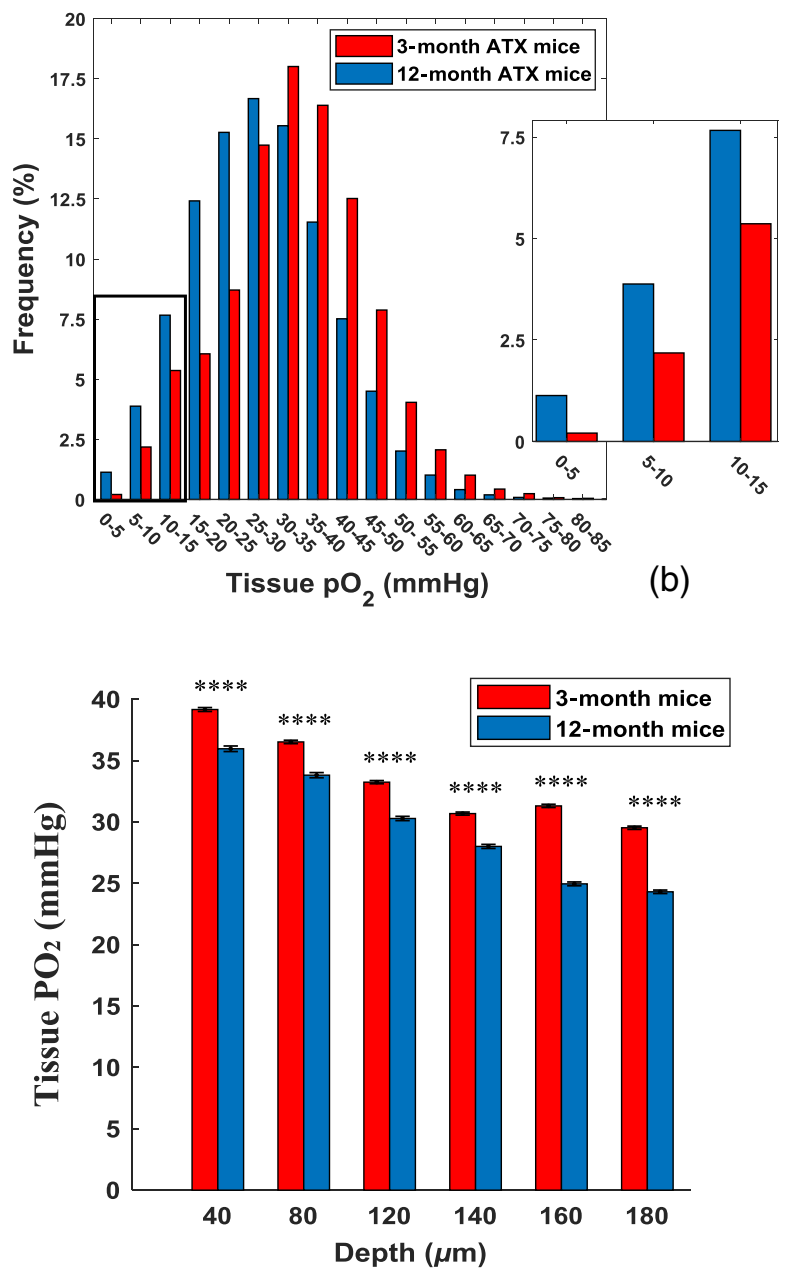

(e)

Fig. 3 Cerebral tissue $\mathrm{PO}_{2}$ measurement with two-photon phosphorescence lifetime imaging. (a1) A typical $\mathrm{PO}_{2}$ grid (20 pixels $\times 20$ pixels) covering an ROI of $400 \mu \mathrm{m} \times 400 \mu \mathrm{m}$. The scale bar is $100 \mu \mathrm{m}$. (a2) The interpolated $\mathrm{PO}_{2}$ image of the $\mathrm{PO}_{2}$ grid (a1). A hypoxic micropocket was identified and delimited by the red lines. (b) The distribution of cerebral tissue $\mathrm{PO}_{2}$ for the two groups of ATX mice. The $\mathrm{PO}_{2}$ distribution of old ATX mice was shifted toward lower $\mathrm{PO}_{2}$ values when compared with the young ATX group. The inset shows that pixels with low $\mathrm{PO}_{2}$ values were more frequently seen in old ATX mice than in young ATX mice. (c) Cerebral tissue $\mathrm{PO}_{2}$ value in old ATX mice was significantly smaller than that in young ATX mice. (d) The old ATX mice showed a significantly higher spatial heterogeneity of tissue $\mathrm{PO}_{2}$ when compared with the young ATX group. The spatial heterogeneity was calculated for each $\mathrm{PO}_{2}$ grid and was defined as the coefficient of variation of each $\mathrm{PO}_{2}$ grid. (e) Tissue $\mathrm{PO}_{2}$ values at different depths were analyzed for the two groups of ATX mice. The decreasing trend of $\mathrm{PO}_{2}$ relative to depth was found in both ATX groups. The old ATX group had significantly lower cortical tissue $\mathrm{PO}_{2}$ at all investigated depths than the young ATX group. Data are shown as mean \pm SEM. Unpaired $t-$ test was performed: ${ }^{* * *} p<0.0001$. Y, young and O, old.

capillary endothelium as discovered in a previous ex vivo study. ${ }^{28}$ In the current work, we were interested in the structural and functional changes of cerebral capillaries in an in vivo proatherogenic environment. Using two-photon fluorescence intensity imaging, reference 2-D images were obtained from which multiple capillaries were selected for subsequent two-photon line scans [Figs. 4(a)-4(b)]. From the perpendicular and longitudinal spatiotemporal line-scan images, we were able to retrieve diameter, $\mathrm{RBC}$ flux, $\mathrm{RBC}$ velocity, $\mathrm{RBC}$ velocity heterogeneity, and hematocrit for each capillary. Comparison of these capillary parameters between the young and the old ATX group is shown in Figs. 4(c)-4(g). Changes in all parameters were statistically significant. RBC flux, RBC speed, and hematocrit of the old ATX mice decreased significantly, whereas the value of capillary diameter and $\mathrm{RBC}$ velocity heterogeneity were found significantly greater compared with young ATX mice.

\section{Discussion}

As an organ consuming a high quantity of oxygen and energy substrates, the brain is vulnerable to hypoxia. The adequate oxygen supply to cerebral tissue and its temporal and spatial regulation relies on a well-functioning cerebral vasculature. In the current work, we investigated the detrimental effects of atherosclerotic disease on cerebral microvasculature and cerebral tissue oxygenation using $\mathrm{LDLR}^{-/-} \mathrm{hApoB}^{+/+}$transgenic mice at 


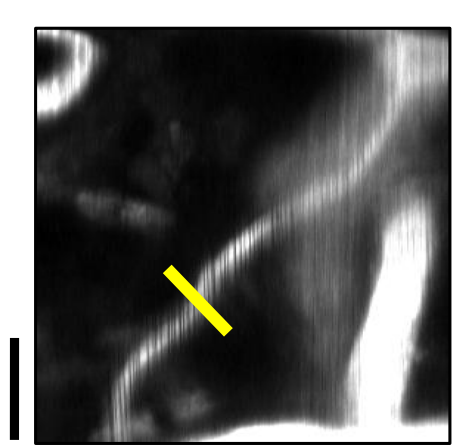

(a1)

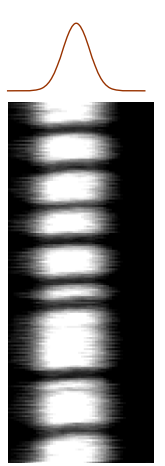

(a2)

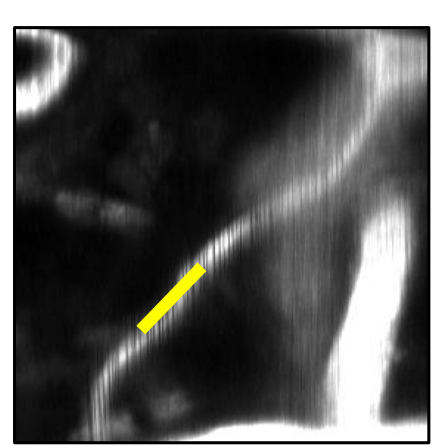

(b1)

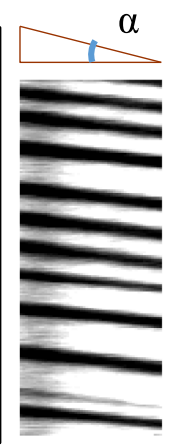

(b2)

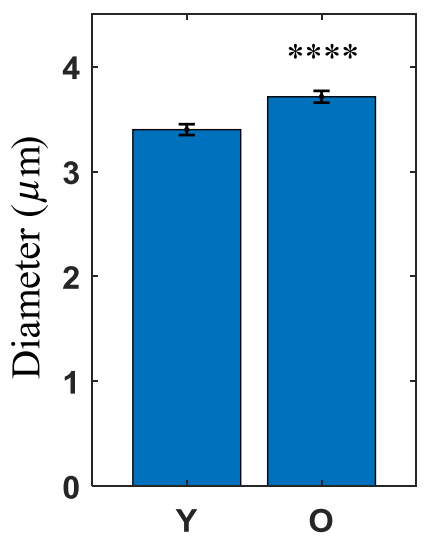

(c)

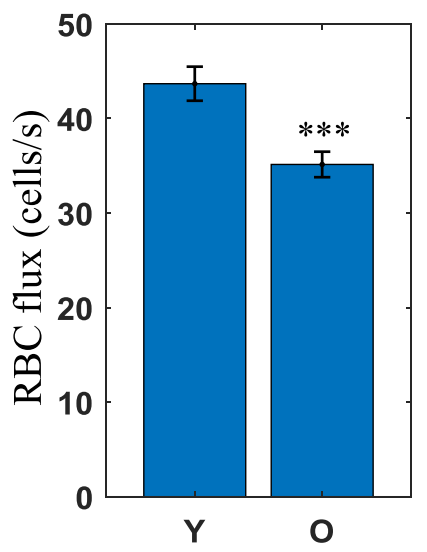

(d)

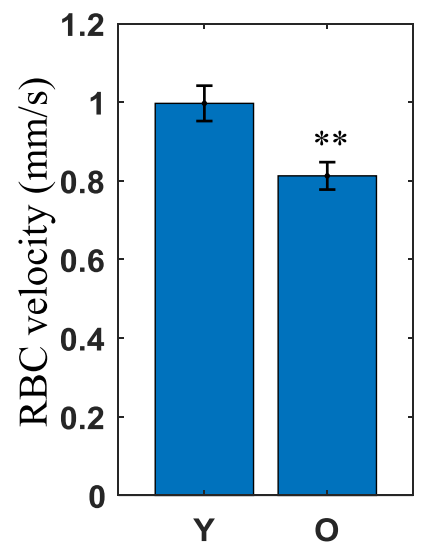

(e)

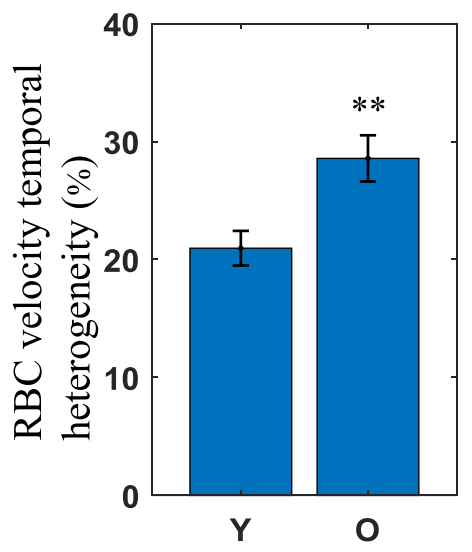

(f)

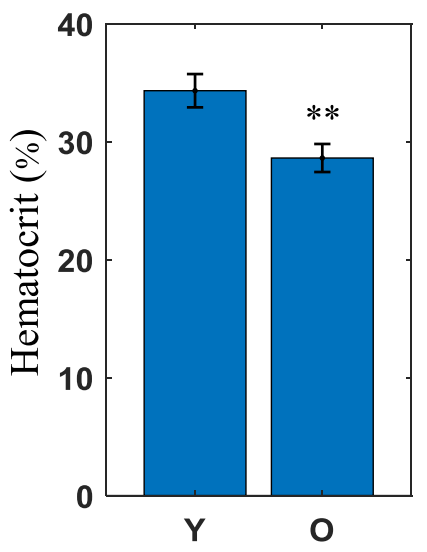

(g)

Fig. 4 Two-photon fluorescence line scans to measure capillary parameters. (a1), (b1) A two-photon fluorescence intensity image in the $x-y$ plane with vessels appearing bright. The yellow line represents the laser scanning direction. Perpendicular line scans were perpendicular to capillaries, and longitudinal line scans were parallel to capillaries. The scale bar is $25 \mu \mathrm{m}$. (a2) A spatiotemporal image composed of 200 consecutive perpendicular scans. A Gaussian function was fitted to the intensity profile averaged from the 200 perpendicular scans to estimate the capillary lumen diameter. (b2) A spatiotemporal image of longitudinal scans. The tilted dark streaks were due to the passage of RBCs in the capillary. RBC speed was calculated from the slope of the streaks, namely the tangent of the angle $\alpha$. (c)-(g) Comparison of the capillary parameters, including diameter, RBC flux, RBC velocity, RBC velocity heterogeneity, and hematocrit, between the young and the old ATX mice. RBC velocity heterogeneity is defined as the coefficient of variation of RBC velocity. RBC flux, RBC speed, and hematocrit of old ATX mice were significantly lower when compared with young ATX mice. The values of capillary diameter and RBC velocity heterogeneity of old ATX mice were significantly higher than those of young ATX mice. Data are shown as mean \pm SEM. Unpaired $t$-test was performed: ${ }^{* \star * *} p<0.0001,{ }^{* * *} p<0.001$, and ${ }^{* \star} p<0.01$. $\mathrm{Y}$, young and $\mathrm{O}$, old.

3 months of age, where no atherosclerotic lesions are found, and old mice at 12 months of age, where atherosclerosis is fully developed. Four optical imaging techniques were employed to characterize the microvascular environment: (1) ISOI for monitoring hemodynamic response in somatosensory cortex to whisker stimulation; (2) Doppler OCT for estimating arteriolar and venular size and blood flow; (3) two-photon phosphorescence lifetime imaging for measuring cerebral tissue $\mathrm{PO}_{2}$; and (4) two-photon fluorescence line scans to investigate capillaries. We used the thinned-skull window approach instead of open-skull technique to reduce inflammation and hemorrhage. As isoflurane, a commonly used anesthetic agent in animal studies for repeated experiments, modulates neural activity and has a vasodilator effect, ${ }^{41,42}$ all our experiments were conducted with awake mice to avoid associated confounds. During imaging sessions, resting states (of the order of minutes) and short bouts of locomotion (of the order of seconds) of the mice were observed to be interleaved. We monitored animals during imaging acquisition. OCT and two-photon images were acquired while the mice stayed still to avoid locomotion. Their stress and attention levels were, however, not monitored. These factors could have a potential impact on the measured cerebral hemodynamic parameters. As for our ISOI acquisition, whisker stimulation under awake condition caused increased animal locomotion. We took multiple recordings and selected the ones where locomotion was minimized. Furthermore, its effect on hemodynamic response in the barrel cortex has previously been found to be negligible. ${ }^{43}$

Data from ISOI showed that hemodynamic responses in terms of $\mathrm{HbO}_{2}, \mathrm{HbR}$, and $\mathrm{HbT}$ were significantly lower in the old ATX group than in the young ATX group. Group-averaged time series of $\mathrm{HbO}_{2}$ and $\mathrm{HbR}$ concentration changes also confirmed that the old ATX mice had much weaker hemodynamic 
response to whisker deflection than the young group, whereas the difference in time delay between the beginning of stimulation and the response was found to be quite similar, as shown in Fig. 1(f). If we assume that whisker stimulation resulted in comparable increase of oxygen metabolism in the somatosensory cortex in both ATX groups, the smaller increase of $\mathrm{HbO}_{2}$ concentration and HbT concentration in the old ATX mice may indicate a less effective regulatory capacity for cerebral blood flow upon cortical activation. However, since neural activity was not measured in our study, we cannot conclude that the observed cerebral hemodynamic alteration in the 12-month-old ATX mice was completely due to atherosclerotic vascular disease. Since neurodegeneration and cerebrovascular disease are often intertwined, ${ }^{44}$ reduced neural response could also contribute to the decreased hemodynamic response.

Using Doppler OCT, we found that arteriolar blood flow and arteriolar lumen area were highly correlated. The data obtained from the young and the old ATX mice also suggest that the decrease of arteriolar diameter and the deterioration of arteriolar blood flow were associated with the presence of atherosclerosis. As atherosclerosis developed in old ATX mice, their arterioles, the primary sites for blood pressure and blood flow regulation, were affected, reducing their diameter, which is in accordance with the observation from an ex vivo study. ${ }^{45}$ Our analysis of arteriolar diameter versus depth also demonstrated that this reduction occurred not only in the proximal end branching off pial arteries but also in the deeper distal end with smaller lumen diameter. These diseased arterioles slowly lost the ability to supply an adequate quantity of oxygenated blood and to regulate the local blood flow. Drastically decreased oxygen supply to cerebral parenchyma by degenerated arterioles could eventually fail to meet the metabolic requirements of neurons. On the other hand, venules seemed not to be affected by atherosclerosis in terms of lumen size and mean blood flow. The fact that venules stayed intact may be explained by lack of smooth muscle cells in their tunica media and low local hemodynamic load. ${ }^{46,47}$

Oxygen molecules diffuse from arterioles and capillaries to interstitial fluid, and neurons and glial cells extract oxygen directly from interstitial fluid. ${ }^{23}$ The oxygen level in the surrounding media is thus crucial for these nerve and glial cells. Using two-photon microscopy with the phosphorescent dye PtP-C343, we found that the old ATX mice had significantly lower tissue $\mathrm{PO}_{2}$ value and significantly higher tissue $\mathrm{PO}_{2}$ heterogeneity compared with the young ATX mice. This finding indicates that atherosclerosis-related vascular disease modulates the balance between oxygen supply and oxygen consumption, which could lead to hypoxic environment in cerebral tissue and may cause irreversible damages to neurons..$^{22,48,49}$ Even without acute ischemic events, such chronic hypoxia in the brain can have adverse effects on cognition. ${ }^{26,50,51}$ Hypoxic micropockets with extremely low $\mathrm{PO}_{2}$ values $(<5 \mathrm{mmHg})$ were identified in two old ATX mice. However, the direct impact of these hypoxic micropockets on local neurons and glia remains unclear, and the possible causative role in hypoxic micropocket formation played by structural and functional alteration and thrombotic occlusions of nearby microvasculature still needs to be further studied. ${ }^{52}$

Capillaries, as a critical component in oxygen delivery, were investigated using a two-photon fluorescence line-scan technique. The old ATX mice exhibited significant lower RBC flux, RBC speed, and hematocrit compared with the young ATX mice. The decrease of capillary RBC flux and RBC speed in the old ATX mice coincided with the decrease of their arteriolar blood flow. Meanwhile, the old ATX mice showed significantly higher RBC velocity heterogeneity than the young ATX group. A high RBC velocity heterogeneity value suggests a disturbed capillary flow pattern, which has been proven to limit the efficacy of oxygen extraction and impairs oxygen supply to brain tissue. $^{53,54}$ Thus alteration of these capillary properties (RBC flux, RBC velocity, RBC velocity heterogeneity, and hematocrit) along with degeneration of parenchymal arterioles probably resulted in the poor cerebral oxygenation in the old ATX mice. ${ }^{23,55,56}$ Interestingly, capillary diameter of the old ATX group was found to be significantly larger than that of the young ATX group. Our in vivo finding is in concordance with a previous ex vivo study that associated larger capillary lumen diameter with atherosclerosis-susceptible transgenic mice with hyperlipidemia based on histological analysis. ${ }^{57}$ It has been demonstrated that cerebral blood flow is not solely controlled by arterioles, and regulation can also occur at the capillary level by pericytes. ${ }^{58-60}$ The observed capillary dilation in the old ATX mice could thus be a coping mechanism of cerebral microvasculature to partially compensate for oxygen supply decrease in the presence of atherosclerotic vascular disease to meet the metabolic requirements of the brain. In addition, capillary dilation also increases its permeability to solutes, which makes oxygen diffusion into surrounding tissue easier. ${ }^{61}$ Even though atherosclerosis-related capillary dilation may help mitigate cerebral hypoperfusion and hypoxia in the resting state, blood flow regulation capacity at the capillary level could decrease when a large number of neurons are activated by stimulations and a substantial amount of oxygen is needed. Furthermore, capillary dilation in old ATX mice may aggravate BBB disruption and impair its barrier functions, causing microhemorrhages in the cortex. ${ }^{28}$

One of the limitations of our work is that the aging effect was not taken into account in our interpretation when we made comparisons between 3-month-old and 12-month-old ATX mouse groups. Previous studies of behavioral/cognitive tests (social exploration, open field, water maze, and novel object recognition) on WT mice indicated that most of the cognitive functions were preserved in old healthy mice (21 months) compared with young healthy ones ( 3 months) despite the aging process. ${ }^{62}$ However, not showing a significant drop in cognitive functions in old WT mice does not mean that the cerebral vasculature of aged mice remained intact. According to a recent publication, aging does modify capillary functions (RBC flux, RBC speed, and hematocrit) and tissue oxygenation. ${ }^{22}$ But the effects seen here at 12 months were larger and are hypothesized to be due to atherosclerosis. It should also be noted that the two aforementioned studies used two distinct substrains of mice (C57BL/ 6JRccHsd and C57BL/6JOlaHsd). As a result, we cannot conclude that the negative impact was solely attributed to atherosclerosis. Aging, as one of the dominant risk factors for atherosclerotic lesion formation, ${ }^{63}$ may exacerbate cerebral vascular degeneration. Interestingly, the study by Moeini et al. ${ }^{22}$ reported that there were no significant changes on cerebral arteriolar diameter and hemodynamics among young, middleaged, and old WT mice, which suggests that the arteriolar deterioration in 12-month-old ATX mice seen in our study were likely caused by atherosclerotic disease.

\section{Conclusion}

In this paper, we demonstrate that atherosclerotic disease has detrimental effects on cerebral microvasculature and tissue 
oxygenation. Our data show that the decreased hemodynamic response to sensory stimulation is associated with the presence of atherosclerosis. When atherosclerotic disease develops, descending arterioles suffer from structural and functional degeneration, whereas ascending venules remain intact. During the progression of atherosclerosis, capillaries undergo RBC flux decrease and develop a more heterogeneous RBC speed pattern. Blood supply decrease from both arterioles and capillaries leads to cerebral tissue hypoxia. Meanwhile, capillary dilation occurs in old ATX mice, which partially compensates for blood flow decrease caused by atherosclerotic disease.

\section{Disclosures}

The authors declare no competing financial interests.

\section{Acknowledgments}

This work was supported by Canadian Institutes of Health Research (CIHR).

\section{References}

1. R. N. Kalaria and C. Ballard, "Stroke and cognition," Curr. Atheroscler. Rep. 3(4), 334-339 (2001).

2. F. G. van Rooij et al., "Persistent cognitive impairment after transient ischemic attack," Stroke 45(8), 2270-2274 (2014).

3. V. C. T. Mok et al., "Delayed-onset dementia after stroke or transient ischemic attack," Alzheimer's Dementia 12(11), 1167-1176 (2016).

4. C. Haffner, R. Malik, and M. Dichgans, "Genetic factors in cerebral small vessel disease and their impact on stroke and dementia," J. Cereb. Blood Flow Metab. 36(1), 158-171 (2016).

5. M. L. Flaherty et al., "Carotid artery stenosis as a cause of stroke," Neuroepidemiology 40(1), 36-41 (2013).

6. C. Banerjee and M. I. Chimowitz, "Stroke caused by atherosclerosis of the major intracranial arteries," Circ. Res. 120(3), 502-513 (2017).

7. J. F. Arenillas, "Intracranial atherosclerosis: current concepts," Stroke 42(Suppl. 1), S20-S23 (2011).

8. M. S. Ellulu et al., "Atherosclerotic cardiovascular disease: a review of initiators and protective factors," Inflammopharmacology 24(1), 1-10 (2016).

9. N. Koren-Morag et al., "Low- and high-density lipoprotein cholesterol and ischemic cerebrovascular disease," Arch. Intern. Med. 162(9), 993999 (2002).

10. R. Benfante et al., "Elevated serum cholesterol is a risk factor for both coronary heart disease and thromboembolic stroke in Hawaiian Japanese men. Implications of shared risk," Stroke 25(4), 814-820 (1994).

11. E. Lindenstrøm, G. Boysen, and J. Nyboe, "Influence of total cholesterol, high density lipoprotein cholesterol, and triglycerides on risk of cerebrovascular disease: the Copenhagen City Heart Study," BMJ 309(6946), 11-15 (1994).

12. C. Ayata et al., "Hyperlipidemia disrupts cerebrovascular reflexes and worsens ischemic perfusion defect," J. Cereb. Blood Flow Metab. 33(6), 954-962 (2013).

13. V. Schächinger and A. M. Zeiher, "Atherosclerosis-associated endothelial dysfunction," Z. Kardiol. 89(21), IX70-IX74 (2000).

14. M. E. Boesen et al., "A systematic literature review of the effect of carotid atherosclerosis on local vessel stiffness and elasticity," Atherosclerosis 243(1), 211-222 (2015).

15. E. Falk, "Pathogenesis of atherosclerosis," J. Am. Coll. Cardiol. 47(18), C7-C12 (2006)

16. J. M. Wardlaw, C. Smith, and M. Dichgans, "Mechanisms of sporadic cerebral small vessel disease: insights from neuroimaging," Lancet Neurol. 12(5), 483-497 (2013).

17. L. Pantoni, "Cerebral small vessel disease: from pathogenesis and clinical characteristics to therapeutic challenges," Lancet Neurol. 9(7), 689-701 (2010).

18. G. W. Blair et al., "Advanced neuroimaging of cerebral small vessel disease," Curr. Treat. Options Cardiovasc. Med. 19(7), 56 (2017).
19. J. J. Heit and M. Wintermark, "Perfusion computed tomography for the evaluation of acute ischemic stroke," Stroke 47(4), 1153-1158 (2016).

20. M. Desjardins et al., "Multimodal study of the hemodynamic response to hypercapnia in anesthetized aged rats," Neurosci. Lett. 563, 33-37 (2014).

21. C. Zhang et al., "Astrocytic endfoot $\mathrm{Ca}^{2+}$ correlates with parenchymal vessel responses during 4-AP induced epilepsy: An in vivo two-photon lifetime microscopy study," J. Cereb. Blood Flow Metab. 39(2), 260271 (2017).

22. M. Moeini et al., "Compromised microvascular oxygen delivery increases brain tissue vulnerability with age," Sci. Rep. 8(1), 8219 (2018).

23. S. Sakadžić et al., "Large arteriolar component of oxygen delivery implies a safe margin of oxygen supply to cerebral tissue," Nat. Commun. 5(1), 5734 (2014).

24. V. Bolduc et al., "Heart rate-associated mechanical stress impairs carotid but not cerebral artery compliance in dyslipidemic atherosclerotic mice," Am. J. Physiol. Circ. Physiol. 301(5), H2081-H2092 (2011).

25. C. Ramírez et al., "ApoB100/LDLR-/- hypercholesterolaemic mice as a model for mild cognitive impairment and neuronal damage," PLoS One 6(7), e22712 (2011).

26. A. Drouin et al., "Catechin treatment improves cerebrovascular flowmediated dilation and learning abilities in atherosclerotic mice," $\mathrm{Am}$. J. Physiol. Heart Circ. Physiol. 300(3), H1032-H1043 (2011).

27. O. de Montgolfier et al., "High systolic blood pressure induces cerebral microvascular endothelial dysfunction, neurovascular unit damage, and cognitive decline in mice," Hypertension 73(1), 217-228 (2019).

28. O. de Montgolfier et al., "Systolic hypertension-induced neurovascular unit disruption magnifies vascular cognitive impairment in middle-age atherosclerotic LDLr-/-:hApoB+/+ mice," GeroScience (2019).

29. E. M. C. Hillman, "Optical brain imaging in vivo: techniques and applications from animal to man," J. Biomed. Opt. 12(5), 051402 (2007).

30. A. Daugherty, "Mouse models of atherosclerosis," Am. J. Med. Sci. 323(1), 3-10 (2002).

31. D. A. Sanan et al., "Low density lipoprotein receptor-negative mice expressing human apolipoprotein B-100 develop complex atherosclerotic lesions on a chow diet: no accentuation by apolipoprotein(a)," Proc. Natl. Acad. Sci. U. S. A. 95(8), 4544-4549 (1998).

32. L. Powell-Braxton et al., "A mouse model of human familial hypercholesterolemia: markedly elevated low density lipoprotein cholesterol levels and severe atherosclerosis on a low-fat chow diet," Nat. Med. 4(8), 934-938 (1998).

33. A. Drouin et al., "Up-regulation of thromboxane A2 impairs cerebrovascular eNOS function in aging atherosclerotic mice," Pflügers Arch. Eur. J. Physiol. 462(3), 371-383 (2011).

34. B. J. A. Janssen et al., "Effects of anesthetics on systemic hemodynamics in mice," Am. J. Physiol. Circ. Physiol. 287(4), H1618-H1624 (2004).

35. F. Kehl et al., "Isoflurane-induced cerebral hyperemia is partially mediated by nitric oxide and epoxyeicosatrienoic acids in mice in vivo," Anesthesiology 97(6), 1528-1533 (2002).

36. K. Masamoto and I. Kanno, "Anesthesia and the quantitative evaluation of neurovascular coupling," J. Cereb. Blood Flow Metab. 32(7), 12331247 (2012).

37. S. Dubeau et al., "Cerebrovascular hemodynamic correlates of aging in the Lou/c rat: a model of healthy aging," Neuroimage 56(4), 1892-1901 (2011).

38. R. A. Leitgeb et al., "Real-time measurement of in vitro flow by Fourierdomain color Doppler optical coherence tomography," Opt. Lett. 29(2), 171-173 (2004).

39. O. S. Finikova et al., "Oxygen microscopy by two-photon-excited phosphorescence," ChemPhysChem 9(12), 1673-1679 (2008).

40. M. Desjardins et al., "Aging-related differences in cerebral capillary blood flow in anesthetized rats," Neurobiol. Aging 35(8), 1947-1955 (2014).

41. D. A. Schwinn, R. W. McIntyre, and J. G. Reves, "Isoflurane-induced vasodilation: role of the alpha-adrenergic nervous system," Anesth. Analg. 71(5), 451-459 (1990).

42. B. F. Matta et al., "Direct cerebral vasodilatory effects of sevoflurane and isoflurane," Anesthesiol. J. Am. Soc. Anesthesiol. 91(3), 677-680 (1999). 
43. H. Takuwa et al., "Reproducibility and variance of a stimulationinduced hemodynamic response in barrel cortex of awake behaving mice," Brain Res. 1369, 103-111 (2011).

44. The Lancet Neurology, "Vascular disease and neurodegeneration: advancing together," Lancet Neurol. 16(5), 333 (2017).

45. P. Kraft et al., "Hypercholesterolemia induced cerebral small vessel disease," PLoS One 12(8), e0182822 (2017).

46. A. Rudijanto, "The role of vascular smooth muscle cells on the pathogenesis of atherosclerosis," Acta Med. Indones. 39(2), 86-93 (2007).

47. H. Zhang et al., "Artery interposed to vein did not develop atherosclerosis and underwent atrophic remodeling in cholesterol-fed rabbits," Atherosclerosis 177(1), 37-41 (2004).

48. E. Taoufik and L. Probert, "Ischemic neuronal damage," Curr. Pharm. Des. 14(33), 3565-3573 (2008).

49. J. M. Lee et al., "Brain tissue responses to ischemia," J. Clin. Invest. 106(6), 723-731 (2000).

50. B. W. Row, Intermittent Hypoxia and Cognitive Function: Implications from Chronic Animal Models, pp. 51-67, Springer, Boston, Massachusetts (2007).

51. J. L. Bass et al., "The effect of chronic or intermittent hypoxia on cognition in childhood: a review of the evidence," Pediatrics 114(3), 805-816 (2004).

52. J. C. Cruz Hernández et al., "Neutrophil adhesion in brain capillaries reduces cortical blood flow and impairs memory function in Alzheimer's disease mouse models," Nat. Neurosci. 22(3), 413-420 (2019).

53. H. Angleys, L. Østergaard, and S. N. Jespersen, "The effects of capillary transit time heterogeneity $(\mathrm{CTH})$ on brain oxygenation," J. Cereb. Blood Flow Metab. 35(5), 806-817 (2015).

54. A. Lücker et al., "The relation between capillary transit times and hemoglobin saturation heterogeneity. Part 1: theoretical models," Front. Physiol. 9, 420 (2018).

55. A. Lücker et al., "The relative influence of hematocrit and red blood cell velocity on oxygen transport from capillaries to tissue," Microcirculation 24(3), e12337 (2017).

56. D. E. Bragin et al., "Increases in microvascular perfusion and tissue oxygenation via pulsed electromagnetic fields in the healthy rat brain," J. Neurosurg. 122(5), 1239-1247 (2015).

57. Z. Süle et al., "Capillary injury in the ischemic brain of hyperlipidemic, apolipoprotein B-100 transgenic mice," Life Sci. 84(25-26), 935-939 (2009).

58. D. Attwell et al., "Glial and neuronal control of brain blood flow," Nature 468(7321), 232-243 (2010).

59. C. N. Hall et al., "Capillary pericytes regulate cerebral blood flow in health and disease," Nature 508(7494), 55-60 (2014).

60. Y. Itoh and N. Suzuki, "Control of brain capillary blood flow," J. Cereb. Blood Flow Metab. 32(7), 1167-1176 (2012).

61. L. Claesson-Welsh, "Vascular permeability-the essentials," Upsala J. Med. Sci. 120(3), 135-143 (2015).

62. G. Wolf et al., "Differentially severe cognitive effects of compromised cerebral blood flow in aged mice: association with myelin degradation and microglia activation," Front. Aging Neurosci. 9, 191 (2017).
63. T. Head, S. Daunert, and P. J. Goldschmidt-Clermont, "The aging risk and atherosclerosis: a fresh look at arterial homeostasis," Front. Genet. 8, 216 (2017).

Yuankang Lu received his master of science degree in engineering from the Université de Technologie de Compiègne, France, in 2014. $\mathrm{He}$ is currently working on his $\mathrm{PhD}$ at the Laboratoire d'Imagerie Optique et Moléculaire of École Polytechnique de Montréal under the supervision of Professor Frédéric Lesage. His current research interests include optical coherence tomography, multimodal optical imaging, and image processing.

Cong Zhang received her $\mathrm{PhD}$ in electrical engineering from the Ecole Polytechnique de Montreal, Canada, in 2016 and subsequently did a postdoc in the Department of Biology, Brandeis University, USA, in 2018. She is now a postdoc in the Department of Nutrition at the University of Montreal and works at Montreal Heart Institute. Her current research interests focus on studying the vascular pulsatility and cerebral oxygen delivery with optical imaging (intrinsic optical imaging and two-photon microscopy) in animals.

Xuecong Lu received his BEng and his MPhil degrees in electronic and information engineering from Hong Kong Polytechnic University, Kowloon, Hong Kong. He is currently working toward his $\mathrm{PhD}$ in biomedical engineering at the Ecole Polytechnique de Montreal, University of Montreal, Montreal, Canada. His current research interests include biomedical optics, cerebral oxygen delivery and consumption, two-photon microscopy, and neurophotonics.

Mohammad Moeini received his PhD in chemical engineering at McGill University, Canada, and subsequently did a postdoctoral fellowship at the Ecole Polytechnique de Montreal, Canada. He is an assistant professor in the Department of Biomedical Engineering at Amirkabir University of Technology (Tehran Polytechnic), Iran. His research interests include tissue engineering, transport phenomena in soft tissue, and biomedical imaging.

Eric Thorin received his $\mathrm{PhD}$ in pharmacology in France in 1991 before moving for postdoctoral training to the University of Glasgow, Scotland, in 1992/1993 and the University of Vermont, USA, in 1994/ 1995. He has been a scientist (since 1996) at the Montreal Heart Institute and a full professor at the University of Montreal. His interests focus on the impact of aging on the vascular endothelium and on cerebrovascular dysfunctions. He also has experience in drug development as past head of cardiovascular pharmacology at Actelion Pharmaceuticals, Switzerland, in 2001/2002.

Frédéric Lesage is a professor of electrical engineering at the École Polytechnique de Montréal and a director of the Optical and Molecular Imaging Laboratory. He has pursued an interdisciplinary career in applied mathematics, physics, and imaging. His current research activities pertain to the development of innovative imaging techniques for neuronal conditions, involving the analysis of optical signals impervious to physiological background noise, $3-D$ image reconstruction using multimodal instruments, time-domain optical parameter recovery, and multimodal imaging (fMRI-optical and EEG-optical). 